\title{
Left Ventricular Dilatation and Posterior Papillary Muscle Displacement in an Ex Vivo Pulsatile Model of Functional Mitral Regurgitation
}

\author{
Krishaporn Kradangnga, DVM, ${ }^{1,2}$ Eric Monnet, DVM, $\mathrm{PhD}^{1}$ \\ ${ }^{1}$ Department of Clinical Sciences, College of Veterinary Medicine and Biomedical Sciences, Colorado State University, Fort Collins, \\ Colorado, USA; ${ }^{2}$ Department of Veterinary Surgery, Faculty of Veterinary Science, Chulalongkorn University, Bangkok, Thailand
}

\section{ABSTRACT}

Background: Surgical method of choice for functional mitral regurgitation (FMR) is debatable, since recurrence of FMR post-annuloplasty has been reported in a significant number of cases. Developing a pulsatile FMR heart model by left ventricular dilatation can be a favorable option for usage in the primary stages of developing new surgical techniques that adjunctively targets the posterior papillary muscle (PPM) geometry.

Methods: PPM of ex vivo ovine hearts $(\mathrm{N}=22)$ was displaced by three different sizes of patches to induce left ventricular dilatation and FMR. Mitral regurgitation (MR) flow, left ventricular and annular geometry were measured from the dynamic pulsatile flow system before and after patch placement.

Results: Outward displacement of PPM was significantly increased in all patch sizes compared to baseline $(P=.016$, $P=.031$, and $P=.008$ from small to large patch, respectively). Left ventricular volume (LVV) significantly increased from $18.53(15.01-26.03) \mathrm{mL}$ at baseline to $27.5(19.45-42.46) \mathrm{mL}$ after large patch placement $(P=.031)$. However, the small and medium patch groups did not show significant changes in the LVV after patch placement. MR significantly increased $554(185-1,919.3) \mathrm{mL} / \mathrm{min}$ after applying the large patch compared to baseline $(P=.016)$. There were no significant changes from baseline in MR flow after applying the small and medium patch. Application of the large patch produced the highest proportion of FMR heart models $(87.5 \%, P=.031)$.

Conclusion: The large patch ex vivo pulsatile heart model demonstrated outward displacement of the PPM and significantly produced MR flow. This ex vivo pulsatile heart model can be used to facilitate surgical techniques that targets the PPM displacement in FMR patients.

\section{INTRODUCTION}

FMR is defined as regurgitation due to dysfunction of the mitral apparatus with normally structural mitral leaflets causing incomplete closure of the mitral orifice. Remodeling of the left ventricular wall after cardiac ischemic event altered

Received December 6, 2017; accepted April 27, 2018.

Correspondence: Eric Monnet, Department of Clinical Sciences, College of Veterinary Medicine and Biomedical Sciences, Colorado State University, Fort Collins, Colorado, USA (e-mail: Eric.Monnet@colostate.edu). ventricular geometry inducing papillary muscles displacement [Kumanohoso 2003]. Displacement of the papillary muscles exert traction of the leaflets through the chordae causing the leaflets to tether and restricting it from normal coaptation. Other factors proposed to cause FMR are asymmetrical dilatation of mitral annulus and increased left ventricular sphericity [Enriquez-Sarano 2009; Kono 1992; Perloff 1972; Sabbah 1992].

Patients with heart failure carries a worse prognosis in the long term when FMR developed [Kumanohoso 2003]. Mitral annular dilation and the displacement of the PPM are main components for imbalanced forces causing leaflets mal-coaptation. Understanding and assessing the geometry of these components and the limitation of standard ischemic FMR repairs can improve and provide better treatment strategies [Braun 2012]. A way to determine the mechanism of imbalance forces on the leaflets leading to FMR is to use ex vivo heart models. Furthermore, heart models can be used in initial studies of surgical strategies for FMR treatments to lessen the cost and time compared with animal models and clinical studies.

Effective ex vivo heart models that mimic FMR have been reported [Katoh 1999; Richards 2009; Siefert 2013], but none are suitable for studying left ventricular remodeling and PPM

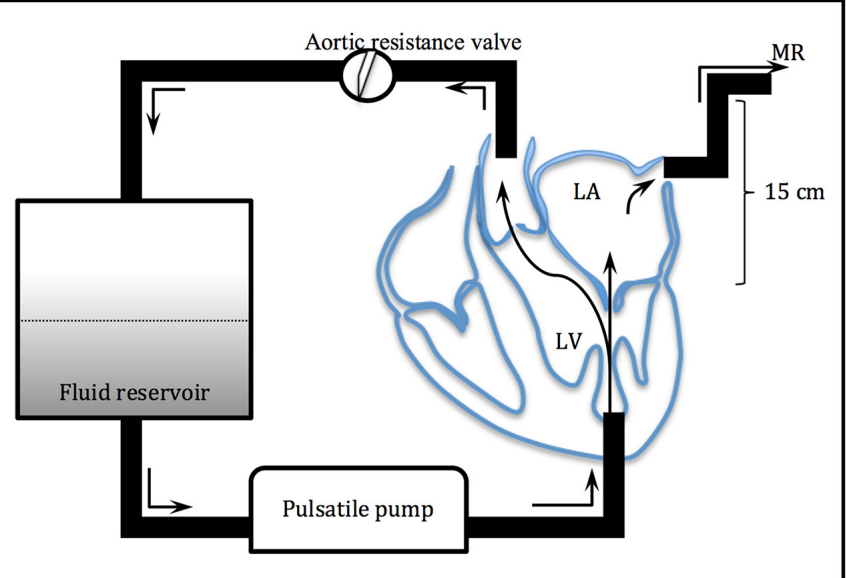

Figure 1. Dynamic pressurization system that generates pulsatile forward flow (indicate as black arrows) through the left ventricle. Mitral regurgitation flow was collected at $15 \mathrm{~cm}$ above the mitral annulus; LA, left atrium; LV, left ventricle; MR, mitral regurgitation. 
Table 1. Left ventricular pressure after patch placement versus baseline in heart models at maximum and minimum pressure. Data is presented as median (IQR).

\begin{tabular}{lcccc}
\hline Patch size & \multicolumn{2}{c}{ Minimum Pressure $(\mathrm{mmHg})$} & \multicolumn{1}{c}{ Maximum Pressure $(\mathrm{mmHg})$} \\
\hline & Baseline & After Patch & Baseline & After Patch \\
Small $(\mathrm{N}=7)$ & $49.7(30.9-51.6) \mathrm{a}$ & $59.2(48.9-64.5) \mathrm{a}$ & $102.5(90.66-118.4) \mathrm{b}$ & $89.3(76.7-100.1) \mathrm{b}$ \\
Medium $(\mathrm{N}=7)$ & $62(54.7-79.5) \mathrm{a}$ & $53.9(47.6-79.5) \mathrm{a}$ & $100.7(96-113.2) \mathrm{b}$ & $97.9(79.3-100.2) \mathrm{b}$ \\
Large $(\mathrm{N}=8)$ & $55.5(40.5-68.8) \mathrm{a}$ & $48.55(20.5-70.85) \mathrm{a}$ & $108.6(95.5-116.05) \mathrm{b}$ & $101.05(79.35-106) \mathrm{b}$ \\
\hline
\end{tabular}

*Minimum and maximum values similar superscripts were no significant difference $(P>.05)$.

displacement. Monnet et al. [Monnet 2013] developed an ex vivo model that has the continuity of the LV wall. Annular dilatation, posterior papillary muscles repositioning or both were used for induction of left ventricular dilatation and FMR. However, the model was built with a static continuous retrograde flow through the aorta.

The purpose of this study is to develop a pulsatile ex vivo model of FMR with a forward flow through the apex of the left ventricle. Determination of the effectiveness of the model and quantifying the MR severity were conducted by measuring MR flow and the geometry of the mitral apparatus when different degrees of PPM displacement were applied to the ex vivo heart model.

\section{MATERIALS AND METHODS}

Fresh ovine hearts were collected from 36 adult Dorsett sheep $70 \pm 5 \mathrm{~kg}$ euthanized for reasons not related to this study, stored at $4^{\circ} \mathrm{C}$. They were prepared for the experiment within 24 hours of euthanasia. Excessive tissue and the pericardium were trimmed out leaving the aorta and pulmonary veins intact. The aorta and left ventricular apex were cannulated with a $3 / 8$-inch cannula. A valve was placed on the cannula coming out of the aorta to control resistance of the output flow. The largest pulmonary vein was cannulated with a $1 / 4$-inch cannula and the rest of the vessels were secured with sutures. The cannula that was placed into the pulmonary vein that had an orifice $15 \mathrm{~cm}$ above the mitral annulus. This was used for measurement of MR flow at $15 \mathrm{~cm}$ of water pressure $(11 \mathrm{mmHg})$. The cannulated aorta and the left ventricular apex were connected to the pulsatile pump system (Figure 1). The pulsatile pump infused the heart with water at room temperature at a rate of 40 cycles per minute with an average flow of $4 \mathrm{~L} / \mathrm{min}$. A pressure transducer (Millar, Houston, Texas, USA) was inserted through the aorta into the left ventricle to measure the left ventricular pressure (LVP). Maximum LVP for each cycle was maintained above $70 \mathrm{mmHg}$ throughout the experiment by adjusting the flow of the pump or resistance in the cannula in the aorta. The pulsatile pump generated a pulsatile flow traveling in a forward flow from the left ventricular apex to the aorta.

To allow displacement of the PPM, a full thickness incision was made in the wall of the left ventricle around the PPM without damaging the chordae. The PPM remains attached to the apex of the left ventricle with a $2 \mathrm{~cm}$ strip of myocardium and to the mitral valve leaflets with the chordae. The left atrium was incised to expose the mitral annulus and leaflets. Under direct visualization, $2 \mathrm{~mm}$ piezo-electric crystals $(2 \mathrm{~mm}$ round Piezo-Electric crystals, Sonometrics, London, Canada) were carefully tunneled into the endocardium based on prior studies on papillary muscles geometry [Green 1999; Gorman 1997; Monnet 2013]. Six 2mm piezo-electric crystals (2mm round Piezo-Electric crystals, Sonometrics, London, Canada) were placed to determine geometry of the annulus and the displacement of the PPM (Figure 2). Patches of diaphragm were sutured around the PPM muscle and the remaining left ventricular wall to reestablish the integrity of the left ventricle. Patches of different sizes were used to induce different degrees of displacement of the PPM. The transducers were connected to a data acquisition system (Sonosoft, Sonometrics, London, Canada).

The hearts were randomly assigned into three different sizes of diaphragmatic patches: Small patch (SP) group, Medium patch (MP) group, and Large patch (LP) group. The patch was $1 \mathrm{~cm}, 2 \mathrm{~cm}$, and $3 \mathrm{~cm}$ at the widest point for the SP, MP, and LP group, respectively. It was sutured with 4-0 suture materials with continuous suture pattern as described by Monnet et al [Monnet 2013]. Distances between each pair of transducers were measured at maximum LVP of each cycle at baseline and after placement of the patch. The MR flow was measured in milliliter per minute $(\mathrm{mL} / \mathrm{min})$. Displacement of the PPM and mitral annular geometry were evaluated at maximum LVP. Hearts with maximum LVP lesser than $70 \mathrm{mmHg}$, abnormalities of the mitral apparatus such as rupture and tear, severe leakage after patch placement, and dislocation of the piezo-electric crystals transducers were excluded from the study.

Data from MR flow and dimension between transducer pairs were expressed as median and interquartile range (IQR) at maximum LVP of the cardiac cycle both at baseline and after patch placement. Comparison of the paired continuous variables in each treatment group from its baseline was performed by Wilcoxon Signed-Rank Test. A Kruskal-Wallis Test was used for comparing continuous variables between treatment groups and comparison of unpaired continuous variables was done by Wilcoxon Rank-Sum Test. Categorical variables comparison was assessed by Fisher's exact test. Significance of the data was considered when $P<.05$. 


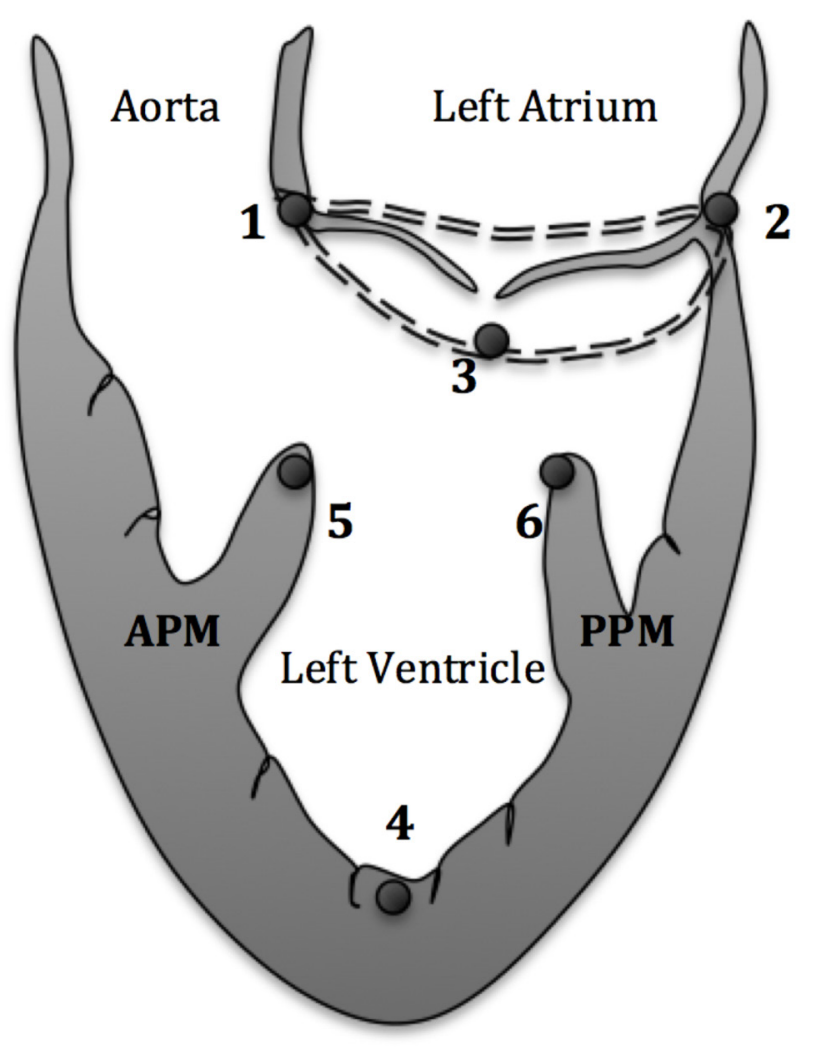

Figure 2. Location of the piezo-electric crystals placement.Piezo-electric crystal No. 1 was placed at the level of the septo-lateral (fibrosa) part of the mitral annulus and piezo-electric crystal No. 2 was placed opposite side of piezo-electric crystal No. 1 on the mid-lateral part of mitral annulus. Piezo-electric crystal No. 3 was placed at the anterior commissure of the mitral annulus and piezo-electric crystal No. 4 at the apex. Piezo-electric crystals No. 5 and 6 were placed on the tip of anterior papillary muscle (APM) and posterior papillary muscle (PPM).

\section{RESULTS}

Thirty-six hearts were used in the study. After placement of the diaphragmatic patches, 22 hearts were included in the final analysis of this study. Fourteen hearts were excluded from the study due to failure to maintain LVP from leakage at suture line (10 hearts), dislodging of the ultrasound transducer (1 heart), and torn left atrium (3 hearts). Seven hearts were in the SP and MP groups and 8 in the LP group. Eleven hearts produced mitral regurgitation: two in each of the SP and MP group; seven in the LP group.

LVP measured at baseline and after patch placement are reported in Table 1 . At baseline and after patch placement, maximum and minimum pressure were not significantly different when compared between the three treatment groups $(P$ $>.05)$. After patch placement, maximum and minimum pressure were not significantly different compared respectively to baseline $(P>.05)$. The maximum LVP for each treatment group was significantly higher than the minimum LVP $(P=$ $.0156,0.0156$ and 0.0078 for SP, MP and LP groups, respectively). The pressure-time relationships before and after
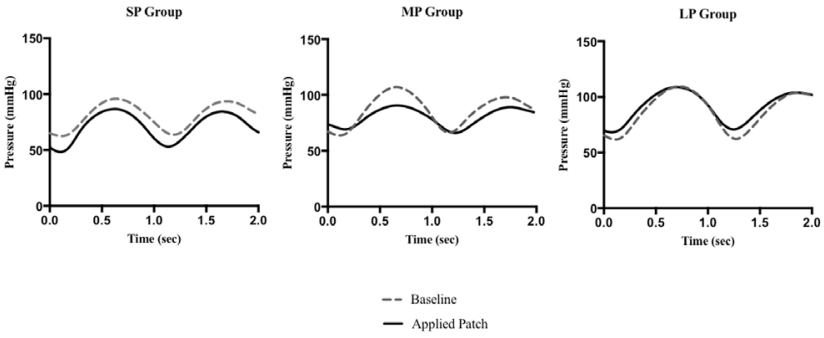

Figure 3. Left ventricular pressure-time graph during one cycle at baseline and after patch application. SP: Small patch; MP; Medium patch; LP; Large patch.

applying the patch generated by the pulsatile pressurization system are illustrated in Figure 3.

Measured MR flow was reported in Table 2. Mitral regurgitation flow increased significantly after applying the large patch from $0 \mathrm{~mL} / \mathrm{min}$ at baseline to $554 \mathrm{~mL} / \mathrm{min}$ (IQR: $185-1,919.3$ $\mathrm{mL} / \mathrm{min}, P=.0156)$. There were no significant changes in the MR flow from baseline in the SP and MP groups $(P>.05)$. In the LP group, 87.5 percent of the hearts had mitral regurgitation while 28.6 percent of the hearts in the SP and MP groups developed mitral regurgitation $(P=.034)$. There was no significant difference between the number of hearts that developed mitral regurgitation between the SP and MP groups $(P>.05)$.

Geometric measurements of the mitral annulus and left ventricle at maximum LVP in the three groups are reported in Table 3. The commissure to commissure annular dimension and mitral annular area were not significantly different after patch placement when compared with baseline in the three groups. In the SP group, after placement of the patch, the distance from fibrosa to PPM $(P=.016)$ and interpapillary length $(P=.016)$ increased significantly from baseline. In the MP group, after placement of the patch, the distance from the fibrosa to $\operatorname{PPM}(P=.031)$, distance from mid-lateral annular to PPM $(P=.031)$ and interpapillary length $(P=.031)$ increased significantly from baseline. In the LP group, after placement of the patch, interpapillary length was significantly increased from baseline $(P=.008)$. Differences of all the dimensions between the three patch sizes were not significant $(P>.05)$. In the LP group, volume of the left ventricle significantly increased from $18.5 \mathrm{ml}$ (IQR: $15.0-26.0 \mathrm{~mL}$ ) at baseline to $27.5 \mathrm{ml}$ (IQR: $19.5-42.5 \mathrm{~mL}$ ) after applying the large patch $(P=.031)$. The LVV did not increase significantly from baseline in the SP and MP groups $(P>.05)$.

\section{DISCUSSION}

Application of a patch around the PPM induced left ventricular dilatation and outward displacement of the PPM. Mitral regurgitation resulted from displacement of the PPM without mitral annular dilatation. This ex vivo model is using a pulsatile forward flow from the apex of the left ventricle toward the aorta.

LVP was maintained during the experiment. Monnet et al. [Monnet 2013] used a large patch sutured around the PPM 
Table 2. Mitral regurgitation flow by patch sizes. Values are shown as Median (IQR)

\begin{tabular}{|c|c|c|c|c|}
\hline Patch Size & Baseline & After Patch & Baseline & After Patch \\
\hline Medium ( $N=7)$ & 0 & $0(0-260)$ & 0 & 2 \\
\hline Large $(\mathrm{N}=8)$ & 0 & $554(185-1,919.3)$ * & 0 & $7 *$ \\
\hline
\end{tabular}

* Indicates significant difference from baseline $(P<.05)$

to induce FMR in a static model of left ventricular remodeling. In this study, after suturing a patch around the PPM a pulsatile flow was used to generate a pressure wave in the left ventricle. The pressure wave was similar before and after placing the patch. A maximum LVP of $100 \mathrm{mmHg}$ was used. The minimum and maximum pressures generated in the left ventricle were not affected by the size of the patch sutured around the PPM. The flow was also maintained in a forward direction from the apex of the left ventricle toward the aorta, which is more physiologic than the direction of the flow used in another study with a continuous flow [Monnet 2013].

Myocardial infarction is commonly associated with asymmetrical left ventricular remodeling with outward displacement of the PPM [Tibayan 2003]. The distance between the fibrosa and the PPM increased as well as distance between the papillary muscles. Therefore, this model reproduces the outward displacement of the PPM reported in several clinical studies [He 1997; Tibayan 2003; Uemura 2005]. This was achieved by suturing a patch only around the PPM. The outward displacement does not seem to correlate with the size of the patch since the distances measured in this experiment were not significantly different between the three different sizes of patches. Left ventricular dilation was only significant with the large patch. In clinical cases, the dilation is the result of an aneurysmal dilation of the infarcted wall around the PPM [He 1997]. Only the larger patch resulted in significant MR and left ventricular dilation. In clinical cases, FMR patients had more dilated annulus and left ventricle compared with normal patients [Enriquez-Sarano 2009; Kumanohoso 2003; Kwan 2003]. Stretching of the mitral valve annulus is possible [Monnet 2013; Richards 2009]. Annular dilation was not induced in this experiment because we wanted to replicate a model reproducing only tethering of the papillary muscle. We wanted a model that would reproduce the left ventricular dilation that is occurring after undersized annuloplasty [Hung 2012; McGee 2004].

Despite claiming rigid annuloplasty as the standard treatment for ischemic FMR, recurrence of moderate to severe MR can be found at 28 percent post-operatively [McGee 2004]. Balanced forces on the leaflets are being explored especially the PPM displacement and the impeding force from the annulus being shifted away from the PPM post-annuloplasty [Capoulade 2016; Jensen 2010; Kalra 2014]. Also having a model reproducing only the outward rotation of the PPM would be more valuable for the evaluation of procedures targeting specifically modification of the geometry of the left ventricle for treatment of FMR.

Mitral valve regurgitation developed mostly with the large patch. The amount of mitral regurgitation was significantly larger with the large patch because the geometry of the left ventricle and the tethering of the PPM on the mitral valve leaflets were more important than with other patches used. The LVV was only significantly affected with the large size patch. The interpapillary muscles distance during maximum LVP increased after application of patches. In vivo studies on FMR have showed similar results [Jensen 2010; Kalra 2014]. Animal models and clinical cases have shown that ischemic FMR was associated with the displacement of the PPM from the septal annulus [Gorman 1997; Tibayan 2003]. Similar measurements were found in the small and medium patch sizes. Variation of the patch sizes was proposed to affect the severity of MR. Although the PPM displacement distances after patch placement were not different between the three patch size groups, the large patch group produced the highest rate of MR and MR volume. This study suggested that the large patch application for the proposed pulsatile ex vivo model of FMR was more appropriate. Larger patch sizes have been used in previous studies and have resulted in larger volume of MR [Monnet 2013] with a patch of $5 \mathrm{~cm}$ at its widest point. The MR was $1383 \mathrm{~mL} / \mathrm{min}$, which was higher than the flow recorded in this present study. Monnet et al. [Monnet 2013] stretched first the mitral annulus contributing to the flow of MR.

\section{LIMITATIONS}

The ex vivo heart used in the pulsatile system were nonvital tissue; therefore the effect of contractility during physiologic cardiac cycle could not be evaluated. To minimize further effects from non-vital tissue, the experiment must be done within 24 hours of the euthanization of the animals. Clinical studies from Otsuji and colleagues [Otsuji 2008] reported that papillary muscles dysfunction or annular dilatation alone were not associate with ischemic FMR. This implied that the pulsatile ex vivo model in this study, despite the absence of contraction, could be used for assessing other factors associated with the MR severity.

In this experiment, many ex vivo constructs were rejected for technical reasons. However, the ex vivo constructs mostly were rejected at the beginning, when we learned how to 
Table 3. Geometric measurements of the annulus and LV chamber after patch placement versus baseline in heart models at maximum pressure. Values are shown as Median (IQR)

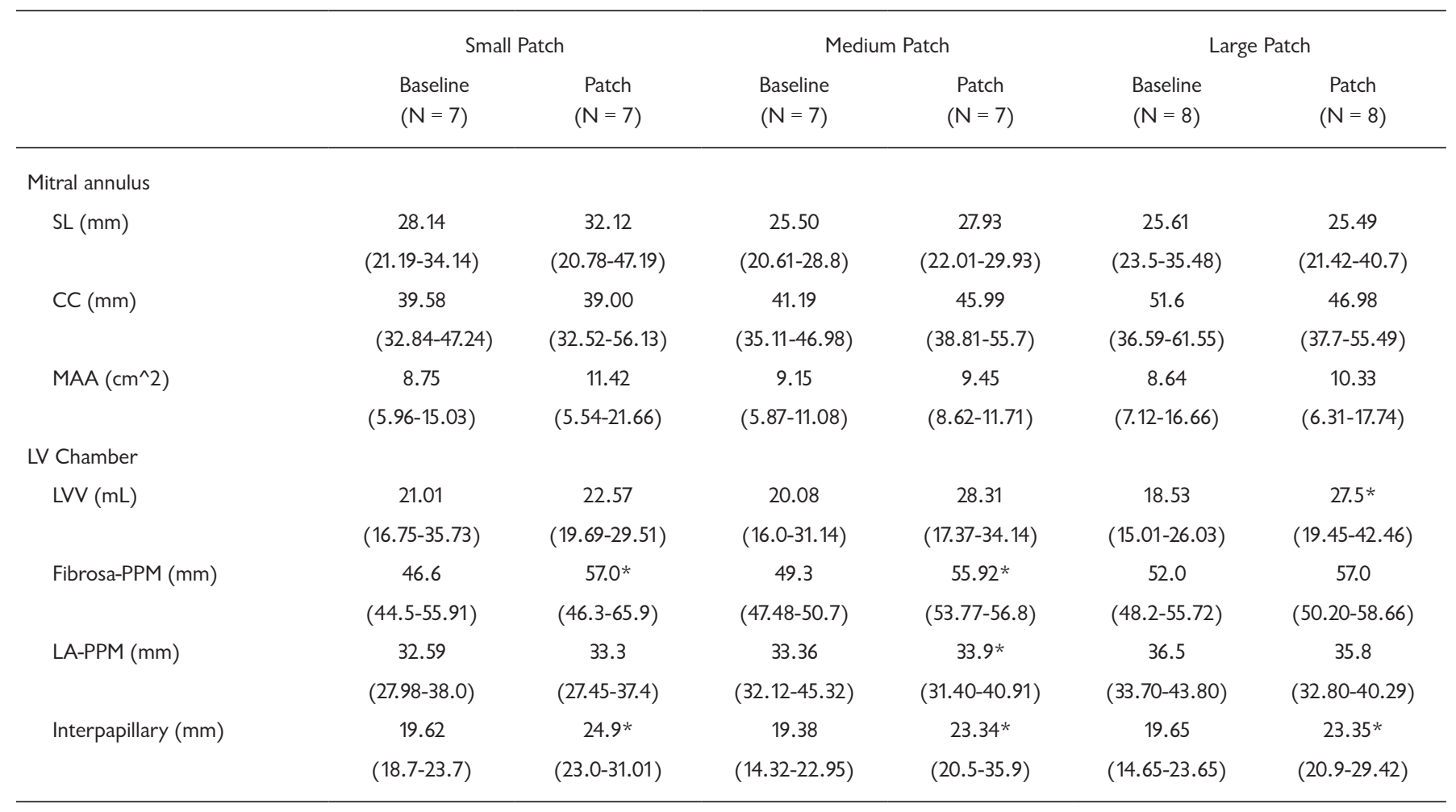

SL: septo-lateral; CC: commissure to commissure; LVV: left ventricular volume; PPM: posterior papillary muscle; LA: lateral annular; *Denotes $P<.05$ versus baseline.

suture the patches on the myocardium. Also, we noticed each construct could be used only for a limited number of experiments because the patches began to tear from the myocardium. The highest maximum LVP of $120 \mathrm{~mm} \mathrm{Hg}$ was used because when the pressure was increased the myocardium tore even more frequently. This limitation on the pressure limited the variation of the left ventricular volume during each cycle.

The results need to be carefully interpreted since the mitral valve in its physiological state closes during the isovolumetric contraction phase, when LVP rises and the left ventricular contraction occurs. If mitral leaflet mal-coaptation occurs, MR will be noticeable during the early and late phase of isovolumetric contraction. MR was less seen during the mid-phase, when LVP reaches its maximum (counter acting the tethered force on the leaflets) [He 1997]. In the pulsatile system, the pump generated maximum LVP, forcing the mitral leaflet to close and the LVV increased from fluid pressurization. Leaflets closed at the same pressurization phase as in the physiological heart except for in vivo, when the LVV decreased from left ventricular contraction.

Patch application mimics left ventricular remodeling which occurs in a three-dimensional fashion. Our study, however, only evaluates the PPM displacement in two dimensions. This may be the explanation for no significant differences of the PPM displacement distances between the patch groups. Studies have shown significant three-dimensional geometric displacements of the PPM and tethered angle of the leaflets [Jensen 2010; Kwan 2003; Tibayan 2003]. Three-dimensional PPM geometry needs to be assessed to give a better idea of how the PPM displacement plays into MR severity.

In conclusion, FMR can be induced in a pulsatile ex vivo model of left ventricular dilatation and PPM displacement. Our model generated LVP waveforms and PPM geometry similar to in vivo studies without disrupting the mitral leaflets and chordae, while the left ventricular wall remained intact. Surgical treatments for FMR that involves annuloplasty, left ventricular wall reshaping and PPM repositioning can be applied to this model prior to in vivo studies.

\section{REFERENCES}

Braun J, Klautz RJM. 2012. Mitral valve surgery in low ejection fraction, severe ischemic mitral regurgitation patients: should we repair them all? Curr Opin Cardiol 27:111--7.

Capoulade R, Zeng X, Overbey JR, et al. 2016. Impact of Left Ventricular to Mitral Valve Ring Mismatch on Recurrent Ischemic Mitral Regurgitation After Ring Annuloplasty. Circulation 134:1247-56.

Enriquez-Sarano M, Akins CW, Vahanian A. 2009. Mitral regurgitation. The Lancet 373:1382-94. 
Gorman III M, Gorman RC, Jackson BM, et al. 1997. Distortions of the Mitral Valve in Acute Ischemic Mitral Regurgitation. Ann Thorac Surg 64:1026-1031.

Green GR, Dagum P, Glasson JR, et al. 1999. Mitral Annular Dilatation and Papillary Muscle Dislocation Without Mitral Regurgitation in Sheep. Circulation 100:II95-II102.

He S, Fontaine AA, Schwammenthal E, Yoganathan AP, Levine RA. 1997. Integrated Mechanism for functional mitral regurgitation. Circulation 96:1826-1834.

Hung J, Solis J, Handschumacher MD, et al. 2012. Persistence of mitral regurgitation following ring annuloplasty: is the papillary muscle outside or inside the ring? J Heart Valve Dis 21(2):218-24.

Jensen H, Jensen MO, Smerup MH, et al. 2010. Three-dimensional assessment of papillary muscle displacement in a porcine model of ischemic mitral regurgitation. J Thorac Cardiovasc Surg 140:1312-8.

Kalra K, Wang Q, McIver BV, et al. 2014. Temporal changes in interpapillary muscle dynamics as an active indicator of mitral valve and left ventricular interaction in ischemic mitral regurgitation. J Am Coll Cardiol 64(18):1867-79.

Katoh T, Ikeda N, Nishi K, et al. 1999. A Newly Designed Adapter for Testing an Ex Vivo Mitral Valve Apparatus. Artificial Organs 23:920-3.

Kono T, Sabbah HN, Rosman H, Alam M, Jafri S, Goldstein S. 1992. Left ventricular shape is the primary determinant of functional mitral regurgitation in heart failure. J Am Coll Cardiol 20:1594-1598.

Kumanohoso T, Otsuji Y, Yoshifuku S, et al. 2003. Mechanism of higher incidence of ischemic mitral regurgitation in patients with inferior myocardial infarction: quantitative analysis of left ventricular and mitral valve geometry in 103 patients with prior myocardial infarction. J Thorac Cardiovasc Surg 125:135-43.

Kwan J, Shiota T, Agler DA, et al. 2003. Geometric Differences of the
Mitral Apparatus Between Ischemic and Dilated Cardiomyopathy With Significant Mitral Regurgitation Real-Time Three-Dimensional Echocardiography Study. Circulation 107:1135-40.

McGee J, Edwin C, Gillinov AM, et al. 2004. Recurrent mitral regurgitation after annuloplasty for functional ischemic mitral regurgitation. J Thorac Cardiovasc Surg 128:916-24.

Monnet E and Pouching K. 2013. An ex vivo model of left ventricular dilation and functional mitral regurgitation to facilitate the development of surgical techniques. The Heart Surgery Forum 16(6): 332-38.

Otsuji Y, Levine RA, Takeuchi M, Sakata R, Tei C. 2008. Mechanism of ischemic mitral regurgitation. J Cardiol 51:145-156.

Perloff JK, Roberts WC. 1972. The mitral apparatus functional anatomy of mitral regurgitation. Circulation 46:227-239.

Richards AL, Cook RC, Bolotin G, Buckner GD. 2009. A Dynamic Heart System to Facilitate the Development of Mitral Valve Repair Techniques. Ann Biomed Eng 37:651-60.

Sabbah HN, Kono T, Rosman H, Jafri S, Stein PD, Goldstein S. 1992. Left ventricular shape: A factor in the etiology of functional mitral regurgitation in heart failure. Am Heart J 123:961-966.

Siefert AW, Rabbah JPM, Koomalsingh KJ, Touchton SA Jr, Saikrishnan N, McGarvey JR. 2013. In Vitro Mitral Valve Simulator Mimics Systolic Valvular Function of Chronic Ischemic Mitral Regurgitation Ovine Model. Ann Thorac Surg 95:825-30.

Tibayan FA, Rodriguez F, Zasio MK, et al. 2003. Geometric Distortions of the Mitral Valvular-Ventricular Complex in Chronic Ischemic Mitral Regurgitation. Circulation 108:II-116-II-121.

Uemura T, Otsuji Y, Nakashiki K, et al. 2005. Papillary muscle dysfunction attenuates ischemic mitral regurgitation in patients with localized basal inferior left ventricular remodeling: insights from tissue Doppler strain imaging. J Am Coll Cardiol 46:113-9. 